\title{
Confinement of a Large Number of Antiprotons and Production of an Ultraslow Antiproton Beam
}

\author{
N. Kuroda, ${ }^{1,2}$ H. A. Torii, ${ }^{1}$ K. Yoshiki Franzen, ${ }^{2, *}$ Z. Wang, ${ }^{1}$ S. Yoneda, ${ }^{1}$ M. Inoue, ${ }^{1}$ M. Hori, ${ }^{3}$ B. Juhász, ${ }^{4}$ D. Horváth, ${ }^{5}$ \\ H. Higaki, ${ }^{6}$ A. Mohri, ${ }^{2}$ J. Eades, ${ }^{7}$ K. Komaki, ${ }^{1}$ and Y. Yamazaki ${ }^{1,2}$ \\ ${ }^{1}$ Institute of Physics, University of Tokyo, Komaba, Meguro-ku, Tokyo 153-8902, Japan \\ ${ }^{2}$ Atomic Physics Laboratory, RIKEN, Wako, Saitama 351-0198, Japan \\ ${ }^{3} \mathrm{CERN}, \mathrm{CH}-1211$ Genève 23, Switzerland \\ ${ }^{4}$ Institute of Nuclear Research of the Hungarian Academy of Science, H-4001 Debrecen, Hungary \\ ${ }^{5}$ KFKI Research Institute of Particle and Nuclear Physics, H-1525 Budapest, Hungary \\ ${ }^{6}$ Plasma Research Center, University of Tsukuba, Tsukuba, Ibaraki 305-8571, Japan \\ ${ }^{7}$ Department of Physics, University of Tokyo, Hongo, Bunkyo-ku, Tokyo 113-0033, Japan
}

(Received 12 May 2003; published 18 January 2005)

\begin{abstract}
We have used a radio frequency quadrupole decelerator to decelerate antiprotons emerging from the CERN Antiproton Decelerator from $\mathrm{MeV}$ - to $\mathrm{keV}$-scale energy, and collected five decelerated pulses in a multiring trap. Some $5 \times 10^{6}$ antiprotons were stacked in this way. Cooling of the trapped antiprotons by a simultaneously trapped electron plasma was studied nondestructively via shifts in plasma mode frequencies. We have also demonstrated the first step in extracting a 10-500 eV antiproton beam from the trap.
\end{abstract}

DOI: 10.1103/PhysRevLett.94.023401

The preparation of large samples of antiprotons at extremely low energy is an important step on the road to synthesizing antihydrogen $\left(\bar{p} e^{+}\right)$and protonium $(\bar{p} p)$ atoms. Not surprisingly, such exotic atoms can only be efficiently synthesized from component particles at the "chemical" energy scale (eV and lower), and this is far below the mandatory $\mathrm{GeV}$ scale of accelerator-produced antiprotons.

Since 2000, this energy gap has been partially bridged by the CERN Antiproton Decelerator (AD), which decelerates antiprotons with $\sim \mathrm{GeV}$-scale kinetic energy to $5.3 \mathrm{MeV}$, and reejects them every $\sim 2 \mathrm{~min}$ in $90 \mathrm{~ns}$ long pulses, containing some $3 \times 10^{7}$ particles. This beam energy is, however, still far above the $10 \mathrm{keV}$ range at which antiprotons can readily be captured and cooled in electromagnetic traps [1-3]. Two groups working at the AD [4-7] have nevertheless demonstrated antihydrogen synthesis in such traps. In these experiments, $\sim 70000 \mu \mathrm{g} / \mathrm{cm}^{2}$ degrader foils reduced the antiproton energy further from the $\mathrm{MeV}$ scale to the $\mathrm{keV}$ scale [1-3]. Many incident antiprotons stop and annihilate within such relatively thick foils, while others emerge with too high an energy to be confined in the potential well of any trap located downstream. Indeed, the largest number of captured particles so far reported for a typical AD pulse, or "shot," is $\sim 2.5 \times$ $10^{4}$ [8]. We describe here the replacement of these simple degrader foils by a radio frequency quadrupole decelerator (RFQD) $[9,10]$. This substantial decelerator, $4 \mathrm{~m}$ in length, reduced the $5.3 \mathrm{MeV}$ AD beam energy between 10 and $120 \mathrm{keV}$. Some $(5-9) \times 10^{6}$ antiprotons within a single shot fulfilled the phase condition necessary for acceptance and deceleration by the RFQD, the remainder being transmitted without deceleration $[9,10]$. We expected that the reduced antiproton energy would allow a much larger number of the emerging antiprotons to be captured in a trap placed after the RFQD than is normally the case for
PACS numbers: 36.10.-k, 39.10.+j, 52.27.Jt, 52.35.Fp

simple degrader foils. For these experiments we used a multiring trap (MRT) comprised of several ring electrodes lying perpendicular to the beam and on the axis of a superconducting solenoid at $10 \mathrm{~K}$, as is shown in Fig. 1.

Since the antiprotons were to spend a long time in this trap, more stringent vacuum conditions were required there to avoid antiproton loss by annihilation on residual gas atoms [3] than in the RFQD, through which they passed only once. Two $90 \mu \mathrm{g} / \mathrm{cm}^{2}$ polyethylenterephtalat (PET) foils located $70 \mathrm{~cm}$ upstream of the MRT were therefore used to isolate the trap and RFQD vacuum systems, at $\lesssim$ $10^{-12}$ mbar and $\sim 10^{-9} \mathrm{mbar}$, respectively. Although these were some 800 times thinner than those used at the entrance of the traps described in Refs. [1-3], antiprotons traversing them unavoidably suffered a finite mean energy loss $(\sim 100 \mathrm{keV})$. The corresponding angular divergence and straggling cause some $30 \%$ of the antiprotons to annihilate on the way through, but this is to be compared with similar losses in [1-3] totaling 99.9\%, for energy loss $5.3 \mathrm{MeV}$. The potential of the entire RFQD could be floated by several tens of kilovolts relative to ground, and we tuned the value of this floating voltage so as to maximize the capture efficiency of the (grounded) MRT. This occurred at an RFQD beam output energy of $110 \mathrm{keV}$.

After passing through the first (upstream) ring, initially biased at ground potential, the antiproton pulse proceeded along the MRT axis to the position of the last one, which being biased at $-10 \mathrm{kV}$, returned it to the first electrode. The trap was closed around the pulse by a switch which changed the bias of this upstream electrode to $-10 \mathrm{kV}$ before the first antiprotons in the pulse reached it on their return trip. By this time, most of the 90 ns long pulse had entered the trap.

Once inside the MRT, the antiprotons were to be decelerated and cooled for extraction as an ultraslow antiproton beam for use in future experiments (see, e.g., [11-13]). 

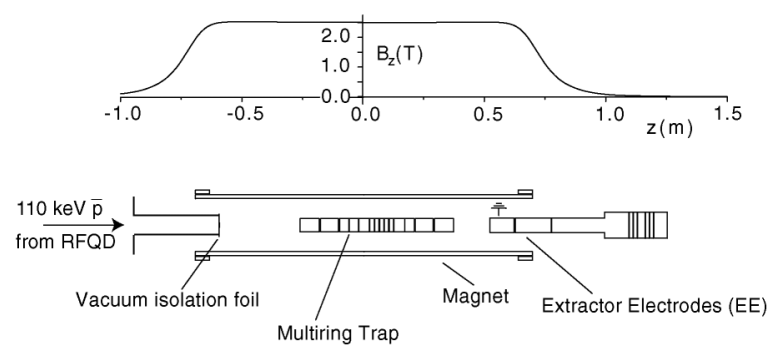

FIG. 1. The MRT and its extractor electrode; the calculated magnetic field distribution $B_{z}$ is also shown.

Before the antiprotons arrived, we therefore loaded the trap with a non-neutral plasma of $\sim 3 \times 10^{8}$ electrons and allowed this to reach equilibrium, where its shape was spheroidal. This plasma cooled the newly arrived antiprotons continuously, since the thermal energy it received from the antiprotons was dissipated spontaneously by synchrotron radiation in the strong magnetic field with a time constant of about $1 \mathrm{~s}[3,14]$. The MRT design enables a large-volume harmonic potential to be established, within which plasmas containing large numbers of charged particles can be stably confined for long periods [15]. Observing the joint plasma modes then allowed us to monitor the plasma shape, density, and temperature in real time [16].

About $\sim 1.2 \times 10^{6}$ among the captured antiprotons could be stored in the MRT for periods of $10 \mathrm{~min}$ or more, although they were usually ejected earlier. This figure is $\sim 50$ times higher than the previous best values obtained with thick degrader foils [8]. With a 30\% RFQD transmission efficiency for the decelerated particles [17] and the aforementioned $70 \%$ efficiency of transmission through the $90 \mu \mathrm{g} / \mathrm{cm}^{2}$ PET foils, the overall antiproton trapping efficiency, defined as the number captured versus the number in the $\mathrm{AD}$ shot, was $\sim 4 \%$. By stacking five $\mathrm{AD}$ shots in sequence [8], $\approx 5 \times 10^{6}$ antiprotons, the largest number of antiprotons ever stored and cooled, were confined in our MRT.

For diagnostic purposes it was important to know the position and time distribution of antiproton annihilations during the capture, storage, and cooling of the AD pulses. Annihilation detectors consisting of two $2 \mathrm{~m}$ long plastic scintillator bars were used for this purpose. They were placed parallel to the trap axis, at distances of 40 and $74 \mathrm{~cm}$ from it, and had cross sections of $4(\mathrm{H}) \times$ $6(\mathrm{~V}) \mathrm{cm}^{2}$, thus subtending overlapping solid angles of $0.12 \mathrm{sr}$ seen from the center of the MRT trap axis. Coincident light pulses produced in these scintillators by the passage of charged particles from nearby antiproton annihilations were recorded by photomultiplier tubes at the ends of the bars, and their arrival-time difference permitted the annihilation vertices to be determined axially with a precision of $\approx 50 \mathrm{~mm}$. We performed particle-tracking simulations using the GEANT library [18], from which we estimate the overall detection efficiency to be $\approx 5 \%$ at the center of the trapping region. With the MRT residual gas pressure of $\approx 10^{-12}$ mbar, most of the annihilations could safely be assumed to occur on the trap cylindrical electrodes (at radius $20 \mathrm{~mm}$ ) or on other adjacent solid materials, such as the vacuum isolation foil.

We excited axially symmetric electrostatic oscillation $(l, 0)$ modes $(l=1-4)$ of the joint plasma by applying white noise to it via one of the trap electrodes. These oscillations induced peaks in the power spectrum at the corresponding mode frequencies, and the shift of these peaks with time was used to monitor the antiproton cooling process. The charge distributions of these modes were axially symmetric and had zero, one, and two nodes along the axial direction, respectively.

Figure 2(a) shows an example of the time evolution of the power spectrum. Resonances corresponding to the $(1,0),(2,0)$, and $(3,0)$ electron plasma modes occur at frequencies 10.6, 17.7, and $22.8 \mathrm{MHz}$, respectively, when undisturbed by the presence of antiprotons in the trap. After the antiprotons were introduced, the electron plasma received energy from them, as a result of which the $(2,0)$ and $(3,0)$ mode frequencies first increased for a few seconds, then decreased with a time constant of a few tens of seconds as thermal equilibrium was reestablished.

To evaluate the temperature variation of the electron plasma from these frequency shifts [19], we consider for each $l$ the dielectric tensor element along the magnetic field, $\epsilon_{3}$. When the plasma temperature $T_{e}$ is sufficiently low to maintain a sharp plasma boundary, $\epsilon_{3}$ is approximately given by

$$
\epsilon_{3} \approx 1-\frac{\omega_{\mathrm{pe}}^{2}}{\omega_{l}^{2}}\left(1+\frac{\kappa_{B} T_{e}}{m} \frac{3 k_{l}^{2}}{\omega_{l}^{2}}\right)
$$

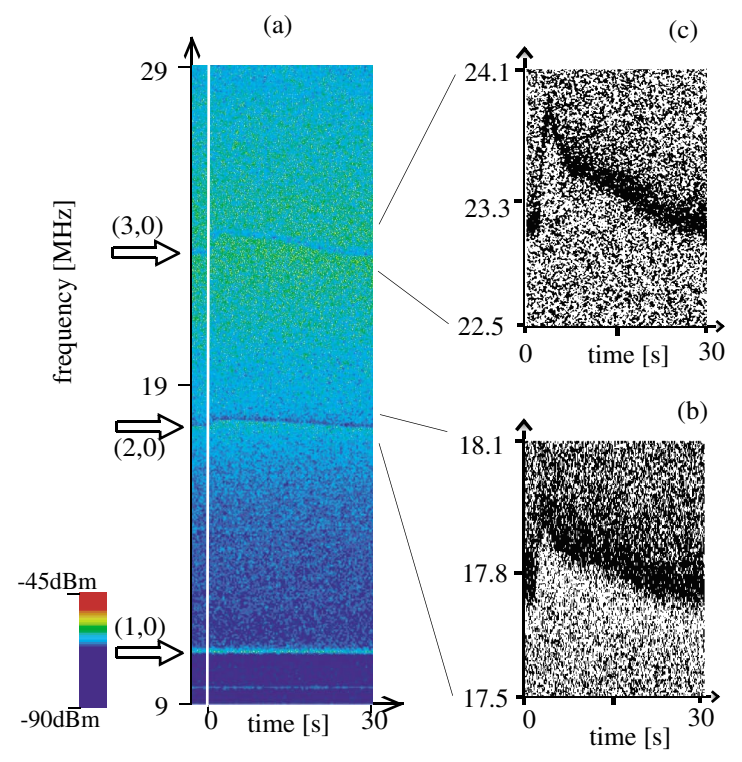

FIG. 2 (color). (a) Time evolution of the power versus frequency spectrum of the electron plasma after antiproton injection at $t=0$. The three arrowed regions correspond to the $(1,0)$, $(2,0)$, and $(3,0)$ modes, respectively; (b) and (c) show the $(2,0)$ and $(3,0)$ modes with expanded vertical scales. 
where $k_{l} \approx \pi(l-1) /(2 b)$ is the longitudinal wave number for mode $(l, 0), 2 b$ the length of the electron plasma, $\kappa_{B}$ the Boltzmann constant, $m$ the electron mass, $\omega_{l}$ the angular frequency of the $(l, 0)$ mode, and $\omega_{\text {pe }}$ the angular frequency of the electron plasma oscillation. The dispersion relation [20] for a cold spheroidal plasma reduces to [19,21]

$$
\epsilon_{3}=\left(\frac{\alpha^{2}-\epsilon_{3}}{\alpha^{2}-1}\right)^{1 / 2} \frac{P_{l}\left(\xi_{1}\right) Q_{l}^{\prime}\left(\xi_{2}\right)}{P_{l}^{\prime}\left(\xi_{1}\right) Q_{l}\left(\xi_{2}\right)}
$$

for a strong magnetic field, where $\xi_{1}=\alpha\left(\alpha^{2}-\epsilon_{3}\right)^{-1 / 2}$, $\xi_{2}=\alpha\left(\alpha^{2}-1\right)^{-1 / 2}$, and $\alpha$ is the aspect ratio, the ratio of the axial length to the diameter of the plasma. $P_{l}$ and $Q_{l}$ represent Legendre functions of the first and second kinds, respectively, while $P_{l}^{\prime}$ and $Q_{l}^{\prime}$ denote their derivatives.

Using the observed $\omega_{l}$ values $(l=2,3$, and 4$)$ before antiproton injection and the electron number previously measured under the same conditions, these equations can be solved to give values $n_{e} \approx 4.4 \times 10^{7} / \mathrm{cm}^{3}$ and $\alpha \approx 7.3$ for the plasma density and the aspect ratio. Using $\alpha$ and $n_{e}$ so obtained, the temperature $T_{e}$ after antiproton injection was evaluated from $\omega_{l}$, which indicated a maximum plasma temperature raise of $\approx 0.6 \mathrm{eV}$. The variations of the plasma frequencies during the cooling that followed heat transfer from the antiprotons were consistently reproduced by solving rate equations which took into account the synchrotron radiation cooling of electrons with time constant $\sim 6 /(B[\mathrm{~T}])^{2}$ s and the energy transfer between the antiprotons and the electron plasma [19]. Considering that the MRT and the bore tube were cooled to $\sim 10 \mathrm{~K}$, the plasma temperature was expected to reach equilibrium at sub $\mathrm{eV}$ energies although no explicit measurements were made.

Figure 3 shows typical antiproton annihilation position distributions as a function of the time elapsed since their injection at $t=0$. The high annihilation rate initially seen at the positions of the trap electrodes and the vacuum isolation foil fell rapidly, leaving about half the captured antiprotons in the MRT after a few seconds. The potential depth of the trap was maintained at $50 \mathrm{~V}$ during a storage period lasting about $55 \mathrm{~s}$. Once the plasma consisting of electrons and antiprotons had stabilized, few additional annihilations were observed until the antiprotons were released.

The antiproton annihilation distributions in Figs. 3(a) and 3(b) refer respectively to cases in which the electron plasma was ejected or retained after its cooling task had been fulfilled. The antiprotons were extracted continuously from the MRT by ramping the trap potential from 50 to $0 \mathrm{~V}$ over a period of some $100 \mathrm{~s}$ started at $t=55 \mathrm{~s}$. In the case shown in Fig. 3(a), we ejected the stored electrons before beginning any antiproton extraction procedure by opening and closing the trap for $550 \mathrm{~ns}$ several times. This was long enough to allow the electrons to escape, but short enough to retain the antiprotons, these having a much lower velocity than the electrons. As expected, no annihilations

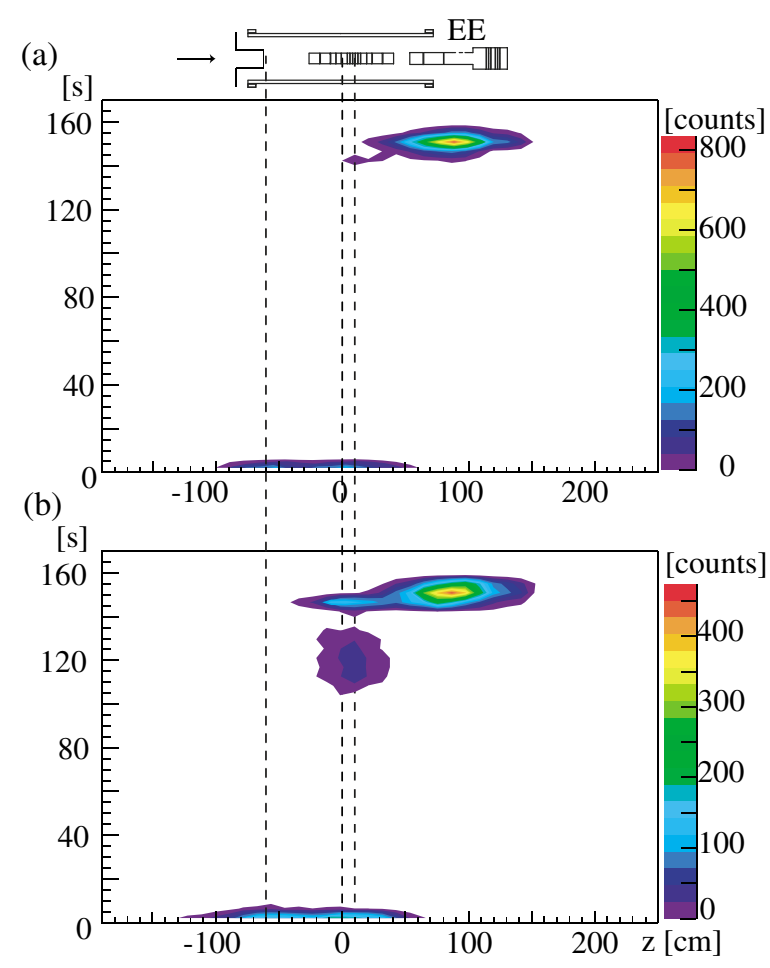

FIG. 3 (color). The $z$ distribution of antiproton annihilations versus the elapsed time since injection at $t=0$ : (a) with and (b) without release of electrons at $40 \mathrm{~s}$ (see text). The superposed drawing shows the vacuum isolation foil at $z=-60 \mathrm{~cm}$, the MRT center at $z=10 \mathrm{~cm}$, and the first part of the beam line containing the extractor electrodes (EE).

were observed during electron ejection at $t \approx 40 \mathrm{~s}$. Furthermore, the fact that none occur between $t=55 \mathrm{~s}$ when ramping started and $t \sim 140 \mathrm{~s}$ confirms that the antiprotons had been cooled into the bottom of the potential well. The strong annihilation peak observed at $t \approx$ 150 s near the downstream extractor electrodes (EE) contained $\sim 90 \%$ of the entire sample of antiprotons trapped in the harmonic potential. Considering that the magnetic field at the EE is $10 \%-20 \%$ of that around the MRT and the inner diameter of the EE is $50 \mathrm{~mm}$, the diameter of the antiproton cloud in the MRT was estimated to be $\sim 15-20 \mathrm{~mm}$.

When [as in Fig. 3(b)] we extracted the antiprotons without first ejecting the electrons, the annihilation pattern changed drastically. The main extraction peak at EE now included only $\sim 75 \%$ of the trapped antiprotons, with the remainder appearing in new peaks at the position of the MRT center, at average times $t \approx 120 \mathrm{~s}$ and $t \approx 145 \mathrm{~s}$. When the first of these new annihilations appeared $(t \approx$ $105 \mathrm{~s}$ ), the depth of trap potential was $\sim 25 \mathrm{~V}$, which is approximately equal to the space charge potential produced by the $3 \times 10^{8}$ electrons contained in the electron plasma. The additional peak can thus be well accounted for by annihilations of antiprotons which hit the electrodes due to the $\mathbf{E} \times \mathbf{B}$ drift caused by instability when electrons overflow from the trap. 
A number of new fields of research would be opened up if the extracted antiprotons could be transported along a beam line to experiments located further downstream. These include collision dynamics in the ionization of atoms by antiprotons [22] and antiprotonic atom formation [13], the study of nuclear surface structure via antiprotonic atom formation and annihilation $[13,23,24]$, as well as the formation of antihydrogen atoms [4-7,25].

We conclude from this work that the combination of the RFQD and the MRT results in as many as $1.2 \times 10^{6}$ cooled antiprotons being accumulated per AD shot; this is at least 50 times larger than the best values reported with thick-foil energy degraders. The cooling feature of antiprotons was studied nondestructively by monitoring electrostatic plasma modes of electrons. A small number of monoenergetic antiprotons of $10-500 \mathrm{eV}$ was then extracted and delivered to a downstream target area via a beam line with differential pumping capacity of more than 6 orders of magnitude. Further studies along these lines will be reported in a future publication.

We express our appreciation to Dr. W. Pirkl, who designed and tested the RFQD, and to the CERN staff for their efforts in providing the antiproton beam. This work was supported by the Grant-in-Aid for Creative Scientific Research (10P0101) of the Japanese Ministry of Education, Culture, Sports, Science and Technology (MonbuKagaku-shō), Special Research Projects for Basic Science of RIKEN, and the Hungarian National Science Foundation (OTKA T033079). Z. W. and K. Y. F. acknowledge their support by JSPS and STA.

*Present address: Department of Physics, University of Florida, Gainesville, FL 32611, USA.

[1] G. Gabrielse et al., Phys. Rev. Lett. 57, 2504 (1986).

[2] M. H. Holzscheiter et al., Phys. Lett. A 214, 279 (1996).

[3] G. Gabrielse et al., Phys. Rev. Lett. 63, 1360 (1989).
[4] M.H. Holzscheiter et al., CERN Reports No. CERN/ SPSLC 96-47 and No. CERN/SPSC P-302, 1996.

[5] G. Gabrielse et al., CERN Reports No. CERN/SPSC 97-8 and No. CERN/SPSC P-306, 1997

[6] M. Amoretti et al., Nature (London) 419, 456 (2002).

[7] ATRAP Collaboration, G. Gabrielse et al., Phys. Rev. Lett. 89, 213401 (2002); 89, 233401 (2002).

[8] G. Gabrielse et al., Phys. Lett. 548B, 140 (2002).

[9] W. Pirkl (private communication); Y. Bylinsky, A. M. Lombardi, and W. Pirkl, hep-ex/0008030; CERN Report No. CERN-PS-2000-061-PP; A. M. Lombardi, W. Pirkl, and Y. Bylinsky, in Proceedings of the 2001 Particle Accelerator Conference, Chicago (IEEE, Piscataway, 2001), p. 585.

[10] M. Hori et al., Phys. Rev. Lett. 91, 123401 (2003).

[11] J. Eades and F. J. Hartman, Rev. Mod. Phys. 71, 373 (1999).

[12] T. Azuma et al., CERN Reports No. CERN/SPSC 97-19 and No. CERN/SPSC P-307, 1997.

[13] Y. Yamazaki, Nucl. Instrum. Methods Phys. Res., Sect. B 154, 174 (1999); Hyperfine Interact. 138, 141 (2001).

[14] B. R. Beck, J. Fajan, and J. Malmberg, Phys. Plasmas 3, 1250 (1996).

[15] A. Mohri et al., Jpn. J. Appl. Phys. 37, 664 (1998).

[16] H. Higaki and A. Mohri, Phys. Lett. A 235, 504 (1997).

[17] A test experiment with protons supplied from a tandem accelerator at Aarhus University showed the deceleration efficiency was about $40 \%$, which indicates that there is still room for a better tuning of the AD and the RFQD.

[18] CERN Application Software Group, GEANT version 3.21, CERN Program Library Long Writeup W5013, 1994.

[19] H. Higaki et al., Phys. Rev. E 65, 046410 (2002).

[20] D. H. Dubin, Phys. Rev. Lett. 66, 2076 (1991).

[21] M. D. Tinkle et al., Phys. Rev. Lett. 72, 352 (1994).

[22] H. Knudsen and J. F. Reading, Phys. Rep. 212, 107 (1992).

[23] A. Trzińska et al., Phys. Rev. Lett. 87, 082501 (2001).

[24] M. Wada et al., in Non-Neutral Plasma Physics IV, edited by F. Anderegg et al., AIP Conf. Proc. No. 606 (AIP, New York, 2001), p. 625.

[25] A. Mohri and Y. Yamazaki, Europhys. Lett. 63, 207 (2003). 